$1-2020$

Pre-treatment HIV-drug resistance associated with virologic outcome of first-line NNRTI-antiretroviral therapy: A cohort study in Kenya

Ingrid A. Beck

Molly Levine

Christine J. McGrath

Steve Bii

Ross S. Milne

See next page for additional authors

Follow this and additional works at: https://ecommons.aku.edu/eastafrica_fhs_mc_intern_med

Part of the Internal Medicine Commons 


\section{Authors}

Ingrid A. Beck, Molly Levine, Christine J. McGrath, Steve Bii, Ross S. Milne, James M. Kingoo, Isaac So, Nina Andersen, Sandra Dross, and Michael Chung 
Research Paper

\title{
Pre-treatment HIV-drug resistance associated with virologic outcome of first-line NNRTI-antiretroviral therapy: A cohort study in Kenya
}

\author{
Ingrid A. Beck ${ }^{\mathrm{a}}$, Molly Levine ${ }^{\mathrm{a}}$, Christine J. McGrath ${ }^{\mathrm{b}}$, Steve Bii ${ }^{\mathrm{a}}$, Ross S. Milne ${ }^{\mathrm{a}}$, \\ James M. Kingoo ${ }^{\mathrm{b}, \mathrm{c}}$, Isaac So ${ }^{\mathrm{a}}$, Nina Andersen ${ }^{\mathrm{a}}$, Sandra Dross ${ }^{\mathrm{a}, \mathrm{b}}$, Robert W. Coombs ${ }^{\mathrm{d}, \mathrm{e}}$, \\ James Kiarie ${ }^{f}$, Bhavna Chohan ${ }^{\mathrm{b}, \mathrm{g}}$, Samah R. Sakr ${ }^{\mathrm{c}}$, Michael H. Chung, ${ }^{\mathrm{b}, \mathrm{h}}$, Lisa M. Frenkel ${ }^{\mathrm{a}, \mathrm{b}, \mathrm{d}, \mathrm{e}, \mathrm{i}, *}$ \\ ${ }^{a}$ Center for Infectious Diseases Research, Seattle Children's Research Institute, Seattle, WA, United States \\ ${ }^{\mathrm{b}}$ Department of Global Health, University of Washington, Seattle, WA, United States \\ ${ }^{\text {c } C o p t i c ~ H o p e ~ C e n t e r ~ f o r ~ I n f e c t i o u s ~ D i s e a s e s, ~ C o p t i c ~ H o s p i t a l, ~ N a i r o b i, ~ K e n y a ~}$ \\ ${ }^{\mathrm{d}}$ Department of Medicine, University of Washington, Seattle, WA, United States \\ e Department of Laboratory Medicine, University of Washington, Seattle, WA, United States \\ ${ }^{\mathrm{f}}$ Department of Obstetrics and Gynecology, University of Nairobi, Nairobi, Kenya \\ ${ }^{g}$ Kenya Medical Research Institute, Nairobi, Kenya \\ ${ }^{\mathrm{h}}$ Department of Medicine, Aga Khan University, Nairobi, Kenya \\ ${ }^{i}$ Department of Pediatrics, University of Washington, Seattle, WA, United States
}

\section{A R T I C L E I N F O}

\section{Article History:}

Received 4 September 2019

Revised 5 December 2019

Accepted 9 December 2019

Available online 14 January 2020

\section{Keywords:}

Efavirenz

Pre-treatment HIV drug resistance

First-line ART

Virologic failure

NNRTI drug resistance

Minority drug resistant variants

\begin{abstract}
A B S T R A C T
Background: Pre-treatment HIV-drug-resistance (PDR) to WHO-recommended 1st-line non-nucleoside reverse transcriptase inhibitors (NNRTI)-based antiretroviral treatment (ART) is increasing in low-resource communities. We evaluated the risk of PDR on treatment failure if detected at single or multiple codons, at minority $(2-9 \%$ ) or higher ( $\geq 10 \%$ ) frequencies during efavirenz- vs. nevirapine-ART.

Methods: We conducted a pooled analysis across three cohorts of Kenyans initiating 1st-line NNRTI-ART between 2006 and 2014. Mutations K103N, Y181C, G190A, M184V and K65R were detected by an oligonucleotide ligation assay (OLA) and confirmed by Sanger and next-generation sequencing (NGS). PDR was defined as detection of any mutation by OLA when confirmed by NGS. Treatment failure, defined as plasma HIV RNA $\geq 400$ copies/mL at month-12 of ART, was compared by PDR genotypes.

Findings: PDR was detected in 59/1231 (4.8\%) participants. Compared to wild-type genotypes, PDR in participants prescribed nevirapine-ART was associated with increased treatment failure [PDR 69.2\% (27/39) vs. wild-type $10.4 \%(70 / 674) ; p=0.0001]$, whether detected as minority [66.7\% (4/6)] or higher [69.7\% (23/33)] frequencies in an individual's HIV quasispecies ( $p=0.002$ and $p<0.0001$, respectively), or mutations at single [50.0\% (12/24)] or multiple [100.0\% $(15 / 15)]$ codons $(p<0.0001)$. During efavirenz-ART, PDR was also associated with increased virologic failure [PDR 25.0\% (5/20) vs. wild-type 5.0\% (25/498); $p=0.005$ ], but only if detected at multiple drugresistant codons [50.0\% (3/6); $p=0.003]$ or high frequencies PDR [33.3\% (5/15); $p=0.001]$.

Interpretation: The risk that PDR confers for treatment failure varies by number of mutant codons and their frequency in the quasispecies, with a lower risk for efavirenz- compared to nevirapine-based regimens. PDR detection and management could extend the effective use of efavirenz-ART in low-resource settings.

Funding: NIH, PEPFAR.
\end{abstract}

(C) 2019 Published by Elsevier Ltd. This is an open access article under the CC BY-NC-ND license. (http://creativecommons.org/licenses/by-nc-nd/4.0/)

\section{Introduction}

Increased worldwide access to antiretroviral therapy (ART) over the past two decades has diminished deaths due to infection with human immunodeficiency virus type-1 (HIV) in low- and medium-

\footnotetext{
* Corresponding author at: Seattle Children's Research Institute, 307 Westlake Ave N, Seattle, WA 98109, United States.

E-mail addresses: Ifrenkel@u.washington.edu, Ifrenkel@uw.edu (L.M. Frenkel).
}

income countries. In 2010, the WHO recommended 1st-line-ART for HIV-infected adults include either nevirapine (NVP) or efavirenz (EFV) in combination with zidovudine (ZDV) or tenofovir (TDF) and lamivudine or emtricitabine (XTC). Given improved outcomes with EFV- compared to NVP-based ART regimens in multiple observational studies, $[1,2]$ in 2013 , the WHO narrowed their recommendations to EFV+TDF+XTC. A rising prevalence of pre-ART drug resistance (PDR), primarily to non-nucleoside reverse transcriptase inhibitors (NNRTI), [3] raises concern that resistant strains could 


\section{Research in context}

\section{Evidence before this study}

Increasing pretreatment HIV drug resistance (PDR) to nonnucleoside reverse transcriptase inhibitors (NNRTI) has attained prevalences $\geq 10 \%$ in many low- and lower-middle income countries. This led the World Health Organization to recommend that programs switch 1st-line antiretroviral therapy (ART) from efavirenz- to dolutegravir-based ART. Past studies reporting that PDR increases the risk of virologic failure during NNRTI-ART often combined data from nevirapine or efavirenz combined with zidovudine or stavudine and lamivudine. We searched PubMed for articles published in English from 1995 through December 2018 with the terms "nevirapine versus efavirenz" and "first-line ART" OR "initial ART", "pretreatment HIV drug resistance" OR "minority drug resistant variants" OR "low-frequency drug resistant variants" AND "treatment failure" OR "virologic failure", and reviewed data from scientific conferences. A recent trial (ANRS 12249) found that PDR did not increase rates of virologic failure with an efavirenz+tenofovir+emtricitabine regimen, suggesting that PDR has a lesser risk of virologic failure with this regimen.

\section{Added value of this study}

This pooled analysis is unique in examining the risk of PDR in a large group of Kenyans initiating 1st-line nevirapine- or efavirenz-based ART within a single clinic system, and provides novel insights into differences in the risks of specific HIV drug resistance mutations on virologic treatment failure during nevirapine- compared to efavirenz-based ART. We found that tenofovir+lamivudine+efavirenz had superior virologic outcomes compared to nevirapine-based regimens, including among ARV-naïve or ARV-experienced individuals and those with wild-type virus or PDR; and while the most common NNRTI mutation, K103N, when detected as a single mutation increased virologic failure with nevirapine-based-ART, it did not increase virologic failure with tenofovir+lamivudine +efavirenz.

\section{Implications of all the available evidence}

Defining the risk that specific mutant codons confer to tenofovir+lamivudine/emtricitabine (XTC)+efavirenz is relevant for public health officials' interpretation of PDR surveillance data and for clinicians' management of HIV-infected individual's treatment. In spite of rising rates of PDR, use of the tenofovir + XTC+efavirenz combination as 1st-line ART could be effective in settings not ready to adopt implementation of dolutegravirbased ART, in subpopulations where it is contraindicated, or in individuals who do not tolerate dolutegravir-based ART.

compromise the effectiveness of ART programs. To address this concern the WHO recommends surveillance of antiretroviral (ARV)naïve individuals for PDR, and when rates reach $\geq 10 \%$ that ART programs implement either testing for PDR prior to initiating ART to guide the choice of ARV, or a programmatic switch of 1st-line ART from NNRTI-based to a non-NNRTI-based ART regimen [4].

WHO recommendations do not address the implications of specific PDR genotypes on the outcome of EFV+TDF+XTC. The risk conferred by specific genotypes has been ascribed through a combination of in vitro studies and clinical associations [5]. This study of Kenyans initiating 1st-line NNRTI-ART provides novel insights into differences in the risks of PDR on virologic treatment failure during NVP- compared to EFV- based ART, including the risks conferred by specific single and combinations of mutant codons, as well as insights into the risks from PDR detected as minority frequency variants within an individual's HIV quasispecies. Refinements of the risk due to specific mutant codons on $\mathrm{EFV}+\mathrm{TDF}+\mathrm{XTC}$ are relevant to public health officials' interpretation of PDR surveillance data and to clinicians' management of HIV-infected individuals' treatments.

\section{Methods}

\subsection{Study populations}

This analysis includes data from Kenyans qualifying to initiate 1 stline NNRTI-based ART based on local guidelines at Coptic Hope Center's HIV Clinics and who enrolled in studies in 2006, 2010 or 2013/4. The 2006 Cohort included adults randomized to counseling and/or pager interventions to enhance ART-adherence, with specimens retrospectively evaluated for the effect of PDR on virologic outcome [6]. The 2010 Cohort included ARV-naïve or single dose-NVP-experienced women enrolled in an observational study of PDR on virologic outcome [7]. The 2013/4 Cohort included individuals $>2$ years of age enrolled into a randomized-control-trial evaluating testing for PDR to guide the selection of ART regimen on virologic failure (Clinicaltrials. gov NCT01898754) [8]. Review boards for protection of human subjects at participating institutions approved each study.

\subsection{ART regimens and testing of plasma HIV RNA levels and virologic outcome}

Participants received NNRTI-based ART supplied by the President's Emergency Plan for AIDS Relief (PEPFAR). Except individuals with PDR in the 2013/4 study's intervention arm received 2nd-line protease inhibitor (PI)-based ART, and these participants are excluded from this analysis. PEPFAR provided a fixed-dose-combination of stavudine (d4T), lamivudine (3TC) and NVP in 2006; ZDV or d4T, 3TC and NVP or EFV in 2010; and ZDV or TDF, 3TC and NVP or EFV in 2013/4. Demographic and clinical information were collected from medical records, and plasma HIV RNA viral load (VL) tests were performed at enrollment and subsequent time-points, including month-12 of ART. This analysis includes all participants from the three cohorts who initiated NNRTIbased ART and had month-12 virologic outcome, with virologic failure defined as $\mathrm{VL} \geq 400$ copies/mL at month- 12 of ART.

\subsection{HIV drug resistance testing}

Pre-ART plasma (2006 and 2010) and PBMC (2010 and 2013/4) were screened for drug resistance by an oligonucleotide ligation assay (OLA) sensitive to $2 \%$ mutant within a participant's viral population [9]. The OLA detected mutations conferring high-level resistance to NVP/EFV (K103N, Y181C, G190A), 3TC (M184V) and beginning in 2010 to TDF (K65R) using probes optimized for HIV type-1 subtypes A, D and $C$. The OLA also quantified the frequency of these mutations in the individual's HIV quasispecies. The primary analysis only included the plasma OLA results from the 2010 cohort, except when plasma results were not available PBMC results were used ( $n=10 / 169)$. Pre-ART specimens with mutations detected by OLA and/or with virologic failure were evaluated for additional mutations by Sanger consensus sequencing and next-generation-sequencing (NGS) by either 454pyrosequencing [6,9] or Illumina assays [10]. Plasma specimens from the time of virologic failure were genotyped by Sanger sequencing and OLA. To minimize reporting of potential false positive OLA near the limit of detection, mutations detected at low frequencies by OLA ( $<25 \%$ of an individual's HIV population) were included in the primary analysis when confirmed by NGS at a mutant frequency of $\geq 1 \%$, while all mutations detected by OLA, including those not confirmed by NGS, were included in a sensitivity analysis. Sequences are available in 
GenBank (accession \# KF544089-KF544288, KJ395348-KJ395349, MH509760-MH509936, MK512771-MK513407).

\subsection{Statistical analysis}

Comparisons between cohorts and enrollment characteristics were made using Kruskal-Wallis test for continuous variables and as appropriate by Chi-square or Fisher's exact test for proportions. Differences in virologic failure at months- 6 or -8 and month- 12 were compared using Fisher's Exact or Chi-square test, as appropriate. Poisson regression models with robust standard errors were used to determine incidence rate ratios (IRR) and 95\% confidence intervals (CI) to examine characteristics associated with virologic failure at 12-months. We determined a priori to adjust our models for viral load at ART initiation and study cohort due to known associations of these factors with use of NVP vs. EFV-based ART and virologic failure outcomes. Additional covariates associated with virologic failure at $P \leq 0.05$ were included in multivariable analysis. Statistical analyses were performed using Stata 14 (Stata Corporation, College Station, Texas).

This manuscript adheres to STROBE guidelines for cohort studies.

\section{Results}

\subsection{Study population characteristics}

Among the three cohorts, a total of 1385 participants had PDR testing prior to initiating NNRTI-based ART. A total of 1231 (89\%) participants were followed through 12 months of ART and were assessed for virologic failure or suppression during this 12-month time interval. Differences between the three cohorts include: (1) the 2010 Cohort was exclusively women with $\sim 50 \%$ having taken ARV prophylaxis for prevention of mother-to-child transmission
(PMTCT) which was associated with higher prevalence of PDR; (2) the median VL progressively decreased with more recent year of enrollment; (3) the median CD4 count of each more recent cohort increased from 123 to 165 to 235 cells $/ \mu \mathrm{L}$ ( $p=0.0001$ ); and (4) the NNRTI shifted from $100 \%$ of participants prescribed NVP-based ART in 2006 , to $85 \%$ in 2010 , to $35 \%$ in $2013 / 4$, replaced by efavirenz. Similarly, the NRTIs shifted from d4T to ZDV to TDF, all combined with 3TC (Table 1).

\subsection{Pre-antiretroviral-treatment drug resistance (PDR)}

Participants in the 2013/4 Cohort intervention arm were excluded from this study if PDR was detected and participant was prescribed PI-based ART. An additional 9 participants with PDR detected at frequencies $<9 \%$ by OLA (median $2 \%$, range $2-4 \%$ ) were classified as wild-type for the primary analysis as their mutations were not confirmed by NGS.

In the primary analysis PDR was detected by OLA and confirmed by NGS in $59 / 1231(4.8 \%, 95 \% \mathrm{CI}, 3.73$ to 6.14$)$ participants at a median mutant frequency of $83 \%$ (range $2-100 \%$, IQR 16-100\%). Among 1079 ARV-naïve participants the prevalence of PDR was $4.1 \%$ (95\% CI, 3.04 to 5.44 ) with a median mutant frequency of $81 \%$ (range $3-100 \%$, IQR 16-98\%), and among the 144 ARV-experienced, PDR prevalence was higher (9.7\%, 95\% CI, 5.77-15.77; $p<0.0001)$, but the median mutant frequency was similar (93\%; range $2-100 \%$, IQR $20-100 \%$ ). Among the 59 participants with PDR, NNRTI mutations (K103N, Y181C, G190A) were detected in all but one (98.3\%) and NRTI mutations (K65R, M184V) were detected in 14 (23.7\%). Single NNRTI mutations were detected in 37 (62.7\%), with K103N most frequently detected (30/59, $50 \cdot 8 \%$ ), multiple NNRTI mutations were detected in 8 (13.6\%), and multiple NNRTI/NRTI mutations in 13 (22.0\%). A single NRTI mutation was detected in one $(1.7 \%)$ participant.

Table 1

Population characteristics and month-12 virologic outcome among subjects by 1 st-line ARV regimen and pre-ART genotype across three Kenyan cohorts.

\begin{tabular}{|c|c|c|c|c|c|}
\hline Variable & Total & Study 2006 & Study 2010 & Study $2013-2014$ & $P$-value \\
\hline N with month-12 outcome & 1231 & 303 & 169 & $759^{\mathrm{a}}$ & \\
\hline Age (years), median (IQR) & $36(30-44)$ & $36(31-43)$ & $33(29-38)$ & $38(31-45)$ & $0.0001^{\mathrm{b}}$ \\
\hline Female, $N(\%)$ & $870(70.7)$ & $202(66 \cdot 7)$ & $169(100 \cdot 0)$ & $499(65.7)$ & $<0.0001^{\mathrm{e}}$ \\
\hline CD4 count (cells/ $\mu \mathrm{L})$, median (IQR) & $186(102-278)$ & $123(65-180)$ & $165(116-233)$ & $235(132-316)$ & $0.0001^{\mathrm{b}}$ \\
\hline Pre-ART VL $\left(\log _{10} \mathrm{c} / \mathrm{mL}\right)$, median (IQR) & $4.95(4.23-5.55)$ & $5.66(5.22-6.02)$ & $4.90(4.35-5.32)$ & $4.66(3.93-5.20)$ & $0.0001^{\mathrm{b}}$ \\
\hline NVP or EFV-ART, $N(\%)$ & & & & & $<0.0001^{\mathrm{c}}$ \\
\hline NVP-ART & $713(57.9)$ & $303(100.0)$ & $144(85.2)$ & $266(35.0)$ & \\
\hline d4T-3TC & $330 / 713(46.3)$ & $303 / 303(100.0)$ & $27 / 144(18.8)$ & $0 / 266(0.0)$ & \\
\hline ZDV-3TC & $219 / 713(30.7)$ & $0 / 303(0.0)$ & $109 / 144(75.7)$ & $110 / 266(41.4)$ & \\
\hline TDF-3TC & $154 / 713(21.6)$ & $0 / 303(0.0)$ & $8 / 144(5.6)$ & $146 / 266(54.9)$ & \\
\hline ABC-3TC & $10 / 713(1.4)$ & $0 / 303(0.0)$ & $0 / 144(0.0)$ & $10 / 266(3.8)$ & \\
\hline EFV-ART & $518(42.1)$ & $0(0.0)$ & $25(14.8)$ & $493(64.9)$ & \\
\hline TDF-3TC & $486 / 518(93.8)$ & $0(0.0)$ & $1 / 25(4.0)$ & $485 / 493(98.4)$ & \\
\hline $\mathrm{ZDV} / \mathrm{d} 4 \mathrm{~T} / \mathrm{ABC}-3 \mathrm{TC}$ & $32 / 518(6.2)$ & $0(0.0)$ & $24 / 25(96.0)$ & $8 / 493(1.6)$ & \\
\hline PDR at enrollment, $N(\%)$ All participants & $59(4.8)$ & $8(2.6)$ & $17(10.1)$ & $34^{\mathrm{d}}(4.5)$ & $0.0010^{\mathrm{e}}$ \\
\hline ARV-naïve & $44 / 1079(4.1)$ & $8 / 303(2.6)$ & $6 / 97(6.2)$ & $30 / 679^{f}(4.4)$ & $0.234^{\mathrm{e}}$ \\
\hline ARV-experienced ${ }^{g}$ & $14 / 144(9.7)$ & $0 / 0(0.0)$ & $11 / 72(15.3)$ & $3 / 72^{f}(4.2)$ & $0.046^{\mathrm{c}}$ \\
\hline $\mathrm{VF} \geq 400 \mathrm{c} / \mathrm{mL}, N(\%)$ All participants & $127(10.3)$ & $24(7.9)$ & $38(22.5)$ & $65(8.6)$ & $<0.0001^{\mathrm{e}}$ \\
\hline Wild-type & $95 / 1172(8.1)$ & $19 / 295(6.4)$ & $26 / 152(17.1)$ & $50 / 725(6.9)$ & $<0.0001^{\mathrm{e}}$ \\
\hline Mutant & $32 / 59(54.2)$ & $5 / 8(62.5)$ & $12 / 17(70.6)$ & $15 / 34(44.1)$ & $0.178^{e}$ \\
\hline ARV-naïve & $102 / 1079(9.5)$ & $24 / 303(7.9)$ & $17 / 97(17.5)$ & $61 / 679(9.0)$ & $0.015^{\mathrm{e}}$ \\
\hline ARV-experienced & $24 / 144(16.7)$ & $0 / 0(0.0)$ & $21 / 72(29.2)$ & $3 / 72(4.2)$ & $<0.0001^{\mathrm{c}}$ \\
\hline
\end{tabular}

Abbreviations: ARV, antiretroviral; ART, antiretroviral therapy; IQR, interquartile range; VL, plasma HIV RNA level; NVP, nevirapine; EFV, efavirenz; d4T, stavudine; 3TC, lamivudine; ZDV, zidovudine; TDF, tenofovir; ABC, abacavir; PDR, pre-ART drug resistance; VF, virologic failure.

a Excludes 44 subjects who were prescribed 1st-line PI-ART, 35 due to PDR diagnosed by OLA and nine switched to PI-ART due to clinical indications.

b Kruskal Wallis test.

c Fisher's Exact test.

d Total PDR at enrollment of parent study was 8.7\% (70/803) including 36 subjects with drug resistance who were prescribed PI-ART, and excluded from this analysis.

e Chi-square.

f 8 subjects missing drug exposure information.

g ARV-experienced denotes participants receiving ARV prophylaxis for prevention of mother-to-child transmission. 
Table 2

Rates of virologic failure at month-12 of NVP- vs. EFV-ART by OLA/NGS-detected drug resistance at 5 HIV pol RT codons (K65R, K103N, Y181C, M184V and G190A) and other factors at enrollment.

\begin{tabular}{|c|c|c|c|c|c|c|c|c|c|}
\hline \multirow[b]{2}{*}{ Variable } & \multicolumn{4}{|c|}{ NVP-ART } & \multicolumn{4}{|c|}{ EFV-ART } & \multirow{2}{*}{$\begin{array}{l}\text { NVP vs. EFV } \\
p \text {-value }\end{array}$} \\
\hline & $N$ & $N \mathrm{VF}$ & $\% \mathrm{VF}$ & $p$-value ${ }^{a}$ & $N$ & $N \mathrm{VF}$ & $\% \mathrm{VF}$ & $p$-value ${ }^{a}$ & \\
\hline Total participants & 713 & 97 & 13.6 & & 518 & 30 & 5.8 & & $<0.0001^{\mathrm{b}}$ \\
\hline ARV-naïve ${ }^{c}$ & 614 & 76 & 12.4 & & 465 & 26 & 5.6 & & $0.0001^{\mathrm{b}}$ \\
\hline ARV-experienced ${ }^{c}$ & 99 & 21 & 21.2 & & 45 & 3 & 6.7 & & 0.031 \\
\hline Wild-type & 674 & 70 & 10.4 & Reference & 498 & 25 & 5.0 & Reference & 0.0008 \\
\hline ARV-naive & 587 & 56 & 9.5 & & 448 & 21 & 4.7 & & \\
\hline ARV-experienced & 87 & 14 & 16.1 & & 43 & 3 & 7.0 & & \\
\hline Any mutants ( $\geq 2 \%)$ & 39 & 27 & 69.2 & $<0.0001$ & 20 & 5 & 25.0 & 0.0049 & 0.0021 \\
\hline ARV-naive & 27 & 20 & 74.1 & & 17 & 5 & 29.4 & & \\
\hline ARV-experienced & 12 & 7 & 58.3 & & 2 & 0 & 0.0 & & \\
\hline $2-9 \%$ mutant $^{\mathrm{d}}$ & 6 & 4 & 66.7 & 0.0016 & 5 & 0 & 0.0 & 1.000 & NA \\
\hline ARV-naive & 5 & 4 & 80.0 & & 4 & 0 & 0.0 & & \\
\hline ARV-experienced & 1 & 0 & 0.0 & & 0 & 0 & 0.0 & & \\
\hline$\geq 10 \%$ mutant & 33 & 23 & 69.7 & $<0.0001$ & 15 & 5 & 33.3 & 0.0014 & NA \\
\hline ARV-naive & 22 & 16 & 72.7 & & 13 & 5 & 38.5 & & \\
\hline ARV-experienced & 11 & 7 & 63.6 & & 2 & 0 & 0.0 & & \\
\hline OLA (+); CS $(-)$ & 10 & 5 & 50.0 & 0.0023 & 6 & 0 & 0.0 & 1.000 & NA \\
\hline ARV-naive & 8 & 5 & 62.5 & & 4 & 0 & 0.0 & & \\
\hline ARV-experienced & 2 & 0 & 0.0 & & 1 & 0 & 0.0 & & \\
\hline OLA (+); CS $(+)$ & 29 & 22 & 75.9 & $<0.0001$ & 14 & 5 & 35.7 & 0.0007 & NA \\
\hline ARV-naive & 19 & 15 & 78.9 & & 13 & 5 & 38.5 & & \\
\hline ARV-experienced & 10 & 7 & 70.0 & & 1 & 0 & 0.0 & & \\
\hline Single K103N & 20 & 9 & 45.0 & 0.0001 & 10 & 1 & 10.0 & 0.412 & NA \\
\hline Single Y181C, G190A or M184V & 4 & 3 & 75.0 & 0.0044 & 4 & 1 & 25.0 & 0.192 & NA \\
\hline Multiple NNRTI/NRTI & 15 & 15 & 100.0 & $<0.0001$ & 6 & 3 & 50.0 & 0.0028 & NA \\
\hline
\end{tabular}

Abbreviations: NVP, nevirapine; EFV, efavirenz; ART, antiretroviral therapy; VF, virologic failure (plasma HIV RNA $>400 \mathrm{copies} / \mathrm{mL}$ ); ARV, antiretroviral; OLA, oligonucleotide ligation assay; CS, consensus sequencing; NA, not applicable; NNRTI, non-nucleoside reverse transcriptase inhibitor; NRTI, nucleoside reverse transcriptase inhibitor.

a Fisher's exact.

b Chi-square test.

c Excludes 8 participants in the EFV-ART group without data for history of ARV exposure.

d Sensitivity analysis including participants with mutations detected by OLA at frequencies 2-9\% that were not confirmed by NGS: 6/10 (60\%) had VF on NVP-ART vs. $1 / 10(10 \%)$ on EFV-ART, $p=0.057$

Note: NA, not applicable, due to small sample size.

\subsection{PDR and virologic outcome during 12 months of NNRTI-ART}

Virologic failure occurred in 127 of 1231 (10.3\%) participants, and occurred at a greater rate in those prescribed NVP-ART compared to EFV-ART (13.6\% vs. $5.8 \%, p<0.0001)$, including those with wild-type virus, PDR, ARV-naïve and ARV-experienced (Table 2). The rates of virologic failure with NVP combined with 3TC and ZDV, d4T or TDF regimens were all higher than with EFV+3TC+TDF ART $(p<0.0001$; Table A1, Appendix). In multivariable analysis adjusting for cohort, enrollment VL, and NRTI type, participants receiving EFV+3TC+TDF had a lower risk of virologic failure compared to those receiving NVPbased ART regimens (adjusted Incident Rate Ratio, IRR: 0.3, 95\% CI $0.1-0.7 ; p=0.008$ ). The proportion that experienced virologic failure by month- 6 or -8 of ART was higher in those with vs. without PDR [93.7\% (30/32) vs. 62.8\% (54/86), $p=0.0006]$.

When compared to participants with wild-type viruses, those who only harbored minority variants, either $2-9 \%$ by OLA or detected by OLA but missed by Sanger sequencing (median 5\%, range $2-25 \%$, IQR $4-11 \%$ ), had an increased rate of virologic failure when prescribed NVP-ART but not EFV-ART (Table 2). (NOTE: Sensitivity analysis including the 9 participants with mutations at frequencies of $2-9 \%$ by OLA, but not confirmed by NGS showed similar results; participants with minority variants prescribed NVP-ART had significantly higher rate of virologic failure compared to those with WT [60\% (6/10) vs. $10.4 \%(68 / 670), p=0.0002]$, but those with minority variants prescribed EFV-ART had rates of virologic failure similar to WT $[10 \%(1 / 10)$ vs. $5 \%(24 / 493), p=0.402])$. Among participants taking NVP-ART, those with a single K103N or multiple NNRTI/NRTI mutations had higher rates of virologic failure compared to those with no PDR mutations (Table 2). In contrast, among participants taking EFV-ART only those with multiple mutations had increased rates of virologic failure compared to those with wild-type genotype, and those with only $\mathrm{K} 103 \mathrm{~N}$ had rates of virologic failure similar to those with wild-type HIV (Table 2).

An increase in the composite parameter "mutant load" (VL $x$ mutant frequency) was associated with an increased risk of virologic failure among participants with PDR (median mutant load 2021 vs. 35,428 copies $/ \mathrm{mL}, p=0.0032$ ), including those with single Y181C, M184V or G190A mutations, and those with multiple NNRTI/NRTI mutations, but not among those with single K103N mutations (Table 3). Effects of mutant load by drug-regimen could not be evaluated due to insufficient prevalence of specific mutations.

Poisson regression models compared virologic failure among participants treated with NVP- vs. EFV-based-ART adjusting for parameters significantly associated with treatment failure, including, CD4 T-cells counts, pre-ART VL, cohort, and PDR (data not shown). The model that best fit the data (Table A2, Appendix) showed independent effects of VL, PDR, cohort and ART regimen on virologic failure: EFV-based ART was associated with a significantly lower risk for virologic failure (IRR 0.43, 95\% CI 0.28-0.66; $p<0.0001$ ) compared to NVP-based ART; higher VL at enrollment was associated with higher risk of virologic failure (IRR 1.19, 95\% CI 1.09-1.29; $p<0.0001$ ); PDR $\geq 2 \%$ was associated with a 5 -fold increased risk of virologic failure (IRR 5.38, 95\% CI, 3.94-7.35, $p<0.0001$ ); and participation in the 2010 and 2013/14 Cohort with higher IRR for virologic failure (3.26, 95\% CI 1.98-5.35; $p<0.0001$ and 2.42, 95\% CI, 1.46-4.02, $p=0.0010$, respectively). These associations remained with inclusion or exclusion of prior nevirapine (single dose) for prevention of mother-to-child transmission as a covariate in the multivariable model. 
Table 3

Pre-ART mutant load (viral load x greatest mutant frequency) compared in participants with viral suppression vs. virologic failure at month-12 of NNRTI-ART.

\begin{tabular}{|c|c|c|c|c|c|}
\hline \multirow[b]{2}{*}{ Pre-ART mutation } & \multicolumn{2}{|r|}{ Viral suppression } & \multicolumn{2}{|r|}{ Virologic failure } & \multirow[b]{2}{*}{$p$-value } \\
\hline & $N$ & Median (IQR) mutant load copies/mL & $N$ & Median (IQR) mutant load copies/mL & \\
\hline Any mutation $(\geq 2 \%)$ & 27 & $2021(670-73,463)$ & 32 & $35,428(14,480-268,908)$ & 0.0032 \\
\hline Single K103N & 20 & $11,790(1355-135,908)$ & 10 & $23,260(15,683-239,244)$ & 0.202 \\
\hline Single Y181C, G190A or M184V & 4 & $830(428-1325)$ & 4 & $87,391(23,726-259,812)$ & 0.021 \\
\hline Multiple NNRTI/NRTI & 3 & $552(460-961)$ & 18 & $57,289(9966-244,671)$ & 0.016 \\
\hline
\end{tabular}

Abbreviations: NNRTI, non-nucleoside reverse transcriptase inhibitor; NRTI, nucleoside reverse transcriptase inhibitor; ART, antiretroviral therapy; IQR, interquartile range.

\subsection{Drug resistance at virologic failure}

Genotypic Sanger sequencing and OLA of virologic failure specimens was completed for 125 and 126 of 127 participants, respectively. Sanger sequencing revealed wild-type in 27 (21.6\%) and drug resistant mutations (DRM) in 98 (78.5\%). These included 32/127 (25.2\%) participants with PDR. Most with PDR maintained these same DRM at virologic failure (23/32; 71.9\%), either with (18/32; $56.3 \%)$ or without $(5 / 32 ; 15.6 \%)$ additional DRM. Different genotypes were detected in the remainder, either different DRMs (7/32; 21.9\%) or wild-type $(2 / 32 ; 6.3 \%)$. PDR minority variants $(<10 \%)$ by OLA were detected less frequently at virologic failure compared to DRM $\geq 10 \%$ $(40.0 \%$ vs. $90.9 \%, p=0.0013)$. At virologic failure, DRM were detected more frequently among the 32 with PDR compared to the 94 participants with wild-type genotype at enrollment (93.7\% vs. $72 \cdot 3 \%$, $p=0.013$ ); with prevalences shown in Table A3 (Appendix). Prevalence of DRM at virologic failure was not significantly different among participants taking NVP-ART vs. EFV-ART (79.2\% vs. $73.3 \%$, $p=0.615$ ). OLA testing of virologic failure plasma specimens detected DRMs in 104/126 (82.5\%). Among the 125 participants with Sanger sequencing at virologic failure, 24 had resistance mutations to AZT, $\mathrm{d} 4 \mathrm{~T}$ or abacavir at codons not tested by the OLA. In addition, the OLA did not detect resistance to NNRTI in one participant with V106M and $\mathrm{Y} 188 \mathrm{C}$, or resistance to 3TC in four participants with M184I, as these mutations were not interrogated by the OLA. However, OLA of plasma at virologic failure identified all participants with DRM by Sanger sequencing and detected low-level DRM in six additional participants.

\section{Discussion}

This pooled analysis is unique in examining the outcome of PDR in a large group of Africans initiating 1st-line NVP- or EFV-based ART within a single clinic system. Salient observations from the study include that PDR increases the risk of (1) virologic failure within a few months of NNRTI-ART initiation; and increases the risk of virologic failure in association with: (2) increased number of mutant codons; (3) increased frequencies of mutant variants within an individual's HIV quasispecies; and (4) at an increased rate with NVPcompared to EFV-based ART regimens. We observed that (5) EFV $+3 \mathrm{TC}+\mathrm{TDF}$ regimens are associated with increased rates of ART-suppression both among ARV-naïve and -experienced individuals; and (6) that participants with virologic failure by month-12 of ART had multiple NNRTI and NRTI mutations regardless of whether at enrollment they had PDR or wild-type genotype.

While randomized trials have not found EFV-based-ART regimens superior to NVP-based ART, [11] multiple observational studies, $[1,2,12,13]$ in addition to ours, show superior virologic and clinical outcomes with EFV- compared to NVP-based ART. Our study is novel in observing that EFV-based ART was more effective than NVP- based ART in suppressing HIV replication in ARV-naïve individuals with likely TDR.
A notable observation in our study is that participants with only the $\mathrm{K} 103 \mathrm{~N}$ mutation, the most prevalent DRM conferring high-level of resistance to NNRTI [5], had similar rates of virologic failure compared to those with wild-type genotypes when prescribed EFV+3TC + TDF, but increased rates of virologic failure with NVP-based ART. A South African study recently reported that PDR primarily with the single K103N mutation was not associated with increased rates of virologic failure during ART with TDF+FTC+EFV [14]. While the same DRM are associated with HIV resistance to EFV and NVP, EFV is more potent than NVP in studies of wild-type and drug resistant HIV variants tested in vitro [2,15], and TDF is more potent than AZT and d4T [16]. In addition, EFV has a longer plasma half-live (40-55 h) [17] compared to NVP (25-30 h) [18], and therefore, delayed or missed EFV doses would be less likely to result in drug levels falling below the inhibitory concentration. These factors, in addition to improved adherence that one would expect with the use of a fixed-dose combination of EFV+XTC+TDF could all contribute to the superior virologic outcome of this regimen. Single Y181C and G190A mutations appeared to increase virologic failure with NVP-ART more than EFVART, but the prevalence of these mutations was insufficient for statistical comparison. PDR with NRTI mutations K65R and M184V were detected infrequently and, except for one participant with a single M184V, always in combination with NNRTI mutations.

EFV-based-ART in our study appeared more effective than NVPbased ART among those with minority variant DRM, regardless as to whether defined as $<10 \%$ of the HIV quasispecies by OLA or as DRM detectable by OLA and undetectable by Sanger sequencing. In sdNVPexposed women initiating a NVP-based regimen, NNRTI minority variants detected by sensitive assays have been associated with VF [19]. Our 2010 Cohort included women exposed to serial ARV for MTCT who tended to have more minority variants, including NRTI mutations not assessed by OLA, and when these women were compared to ARV-naïve women they had increased rates of $\mathrm{VF}$ when given NVP-based ART [7]. Several studies examining the role of minority variants in treatment outcomes have observed associations with treatment failure during NNRTI-regimens in treatment-naïve [20-25] and treatment-experienced patients [26], while other studies, similar to this study, found no association between minority variants detected in ARV-naive African or European patients with virologic failure of 1st-line EFV-ART [14,27-29] or NVP-ART [30]. Some of the aforementioned studies used allele-specific-PCR to detect K103N alone, or K103N +Y181C [19,20,22,23,26,30]. Other studies using next generation sequencing found that G190A [25], or other NNRTI mutations were more prevalent than K103N or Y181C $[21,24,28]$. It is possible that NNRTI or NRTI mutations not tested for contributed to virologic failure.

Several studies of PDR effects on treatment outcomes of ARTnaïve individuals combined different NNRTI or NRTI regimens in their analyses [21-23,25-29]. A comparison of the risk of minority variants across regimens observed increased virologic failure with EFV +ZDV+3TC but not EFV+TDF+FTC [22], similar to our findings. Combined studies suggest that minority variant NNRTI mutations may vary in the risk of virologic failure by the potency of the regimen, 
specific mutation(s), HIV subtype and/or whether the mutation was transmitted, selected by past NNRTI or was spontaneously generated in the host or as an artifact of PCR and/or sequencing.

The effect of minority variants on the risk of virologic failure has also been associated with the absolute numbers of drug-resistant variants (i.e. mutant load). Using allele-specific-PCR, K103N at levels of $>2000$ copies/mL were associated with virologic failure of EFV-ART [22], others described a dose-dependent risk of virologic failure starting at 10-99 copies/mL [20], and a study using next-generationsequencing suggested drug-resistant variants at $<1000$ copies $/ \mathrm{mL}$ were not associated with virologic failure of 1st-line NNRTI-ART [29]. In our study, participants with single K103N and virologic suppression (mostly taking EFV-based ART) had a median mutant load of 11,790 copies/mL, significantly higher than previously described thresholds for virologic failure. While mutant load might be more predictive of virologic failure than the frequency of drug-resistant variants, the failure threshold for different mutations is likely to be dependent on the potency of the ART regimens.

Compared to Sanger sequencing, the OLA used in this study identified PDR in all but one participant (with K70R) who subsequently experienced virologic failure. In addition, OLA identified all participants with DRM by Sanger sequencing at virologic failure, including several participants with drug resistance not detected by Sanger sequencing. An economical and easy to use point of care OLA kit that assesses NNRTI mutations K103N, Y181C, G190A and NRTI mutations M184V and K65R [31] could be used in resource-limited settings to test patients prior to starting EFV-based ART, at virologic failure to guide the choice of 2nd-line ART, or before switching to dolutegravir-based ART.

Limitations of this observational study include the non-randomized comparison between NVP- and EFV-based ART regimens and differences in inclusion criteria across the three cohorts which increases the potential for residual confounding variables in the analysis. The primary differences between the cohorts that may have affected our findings include the lower median pre-ART plasma RNA viral load and higher median CD4 count in the 2013/4 Cohort, and that improved ART adherence was a study objective and likely higher in the 2006 Cohort in which participants were randomized to interventions aimed at improving ART adherence and were given a fixed dose combination of $\mathrm{d} 4 \mathrm{~T}+3 \mathrm{TC}+\mathrm{NVP}$ [6]. However, in regression analysis controlling for viral load, CD4 counts and cohort, enhanced viral suppression remained associated with EFV-ART. Our 2010 Cohort by design had a higher proportion of women who took single or sequential ARV for PMTCT. These women, with higher rates of PDR by OLA, were more frequently prescribed NVP- compared to EFV-based ART, which could have enhanced the association between minority variants and more virologic failure associated with NVP-ART. Also, fewer participants with PDR received EFV-ART, and because relatively few participants had minority variants by OLA this could have reduced our ability to detect associations with virologic failure. Because Sanger sequencing and/or NGS were performed on pre-ART specimens of participants with virologic failure only, our study could not assess the risk for treatment failure of other PDR mutations not detected by OLA. Lastly, in this study we considered participants with mutations at frequencies $2-9 \%$ by OLA that were not confirmed by NGS as having wild-type genotype. However, rates of virologic failure were similar in participants with OLA mutations, regardless of whether confirmed by NGS (4/12 (33.3\%) vs. 3/9 (33.3\%), $p=1.0)$, and both NGS-confirmed and -unconfirmed minority variants were associated with increased rates of virologic failure compared to those with wild-type virus by OLA. This observation suggests that OLA testing may have been more sensitive than NGS, and by discounting mutations not confirmed by NGS, we slightly inflated the risk of virologic failure among participants analyzed as wild-type by OLA.

In summary, the risk that PDR confers for treatment failure of NNRTI-based ART varies by specific single and combinations of codons, and frequency of mutant variants in the individual's viral quasispecies, with substantially less virologic failure for EFV+3TC+TDF compared to NVP-based regimens. These findings suggest that use of assays to detect and manage PDR could maximize viral suppression and extend the effective use of EFV-based antiretroviral treatment regimens in low-resource settings. Despite rising rates of PDR, effectiveness of EFV $+\mathrm{XTC}+\mathrm{TDF}$ as 1 st-line ART is likely greater than predicted by past studies that included NVP-based ART regimens. Thus, EFV+XTC+TDF may retain efficacy in HIV-infected subpopulations where fixed-dose dolutegravir is not approved or contraindicated, such as infants and children weighing $<30 \mathrm{~kg}$, HIV/TB co-infected individuals, or those who do not tolerate dolutegravir-based ART.

\section{Funding}

The funders of the study had no role in the study design, the collection, analysis and interpretation of data, or writing of the manuscript. The corresponding author had full access to all the study data and had final responsibility for the decision to submit for publication.

\section{Declaration of competing interest}

The authors have no conflicts of interest to declare.

\section{Acknowledgments}

We would like to thank the participants who joined our studies, the staff at the Coptic Hope Centers for providing HIV care, and the NIH Project Officers Joseph Fitzgibbon and Catherine Godfrey for helpful suggestions. Viral load tests and sequence analysis were performed with assistance of the Clinical and Retrovirology Research Core and the Molecular Profiling and Computational Biology Core of the UW CFAR [P30 AI027757].

\section{Funding}

This work was supported by grants from the National Institutes of Health awards [R01 AI058723, R01 AI100037 and R01 AI100037 Administrative Supplement to LMF], including an American Recovery and Reinvestment Act supplement [R01 AI058723]. The Coptic Hope Center for Infectious Diseases is supported by the President's Emergency Plan for AIDS Relief through a cooperative agreement [U62/CCU024512 to $\mathrm{MHC}$ ] from the Centers for Disease Control and Prevention.

\section{Supplementary materials}

Supplementary material associated with this article can be found in the online version at doi:10.1016/j.eclinm.2019.100239.

\section{References}

[1] Cain LE, Phillips A, Lodi S, Sabin C, Bansi L, Justice A, et al. The effect of efavirenz versus nevirapine-containing regimens on immunologic, virologic and clinical outcomes in a prospective observational study. AIDS 2012;26(13):1691-705.

[2] Tang MW, Kanki PJ, Shafer RW. A review of the virological efficacy of the 4 world health organization-recommended tenofovir-containing regimens for initial HIV therapy. Clin Infect Dis 2012;54(6):862-75.

[3] Gupta RK, Gregson J, Parkin N, Haile-Selassie H, Tanuri A, Andrade Forero L, et al. HIV-1 drug resistance before initiation or re-initiation of first-line antiretrovira therapy in low-income and middle-income countries: a systematic review and meta-regression analysis. Lancet Infect Dis 2018;18(3):346-55.

[4] World Health Organization. Guidelines on the public health response to pretreatment HIV drug resistance. Geneva, Switzerland: World Health Organization; 2017.

[5] Rhee SY, Gonzales MJ, Kantor R, Betts BJ, Ravela J, Shafer RW. Human immunodeficiency virus reverse transcriptase and protease sequence database. Nucleic Acids Res 2003:31(1):298-303.

[6] Chung MH, Beck IA, Dross S, Tapia K, Kiarie JN, Richardson BA, et al. Oligonucleotide ligation assay detects HIV drug resistance associated with virologic failure among antiretroviral-naive adults in Kenya. J Acquir Immune Defic Syndr 2014;67(3):246-53. 
[7] Milne RS, Silverman RA, Beck IA, McKernan-Mullin J, Deng W, Sibley TR, et al Minority and majority pretreatment HIV-1 drug resistance associated with failure of first-line nonnucleoside reverse-transcriptase inhibitor antiretroviral therapy in Kenyan women. AIDS 2019;33(6):941-51.

[8] Chung MH, McGrath MJ, Beck IA, Levine M, Milne RS, So I, et al. Evaluation of the management of pretreatment HIV drug resistance by oligonucleotide ligation assay: a randomised controlled trial. Lancet HIV 2019. doi: 10.1016/S2352-3018 (19)30337-6.

[9] Beck IA, Deng W, Payant R, Hall R, Bumgarner RE, Mullins JI, et al. Validation of an oligonucleotide ligation assay for quantification of human immunodeficiency virus type 1 drug-resistant mutants by use of massively parallel sequencing. J Clin Microbiol 2014;52(7):2320-7.

[10] Silverman RA, Beck IA, Kiptinness C, Levine M, Milne R, McGrath CJ, et al. Prevalence of pre-antiretroviral-treatment drug resistance by gender, age, and other factors in HIV-Infected individuals initiating therapy in Kenya, 2013-2014. J Infect Dis 2017;216(12):1569-78.

[11] Mbuagbaw L, Mursleen S, Irlam JH, Spaulding AB, Rutherford GW, Siegfried N. Efavirenz or nevirapine in three-drug combination therapy with two nucleoside or nucleotide-reverse transcriptase inhibitors for initial treatment of HIV infection in antiretroviral-naive individuals. Cochrane Database Syst Rev 2016;12: CD004246.

[12] Pillay P, Ford N, Shubber Z, Ferrand RA. Outcomes for efavirenz versus nevirapinecontaining regimens for treatment of HIV-1 infection: a systematic review and meta-analysis. PLoS ONE 2013;8(7):e68995.

[13] Shearer K, Brennan AT, Maskew M, Long L, Berhanu R, Sanne I, et al. The relation between efavirenz versus nevirapine and virologic failure in Johannesburg, South Africa. J Int AIDS Soc 2014;17:19065.

[14] Derache A, Iwuji CC, Baisley K, Danaviah S, Marcelin AG, Calvez V, et al. Impact of next generation sequencing defined HIV pre-treatment drug resistance on virological outcomes in the ANRS 12249 treatment as prevention trial. Clin Infect Dis 2018;69(2):207-14.

[15] Sluis-Cremer N, Tachedjian G. Mechanisms of inhibition of hiv replication by nonnucleoside reverse transcriptase inhibitors. Virus Res 2008;134(1-2):147-56.

[16] Louie M, Hogan C, Hurley A, Simon V, Chung C, Padte N, et al. Determining the antiviral activity of tenofovir disoproxil fumarate in treatment-naive chronically HIV-1-infected individuals. AIDS 2003;17(8):1151-6.

[17] Sustiva package insert. 2017. https://packageinserts.bms.com/pi/pi_sustiva.pdf.

[18] Havlir D, Cheeseman SH, McLaughlin M, Murphy R, Erice A, Spector SA, et al. High-dose nevirapine: safety, pharmacokinetics, and antiviral effect in patients with human immunodeficiency virus infection. J Infect Dis 1995;171(3):537-45.

[19] Boltz VF, Zheng Y, Lockman S, Hong F, Halvas EK, McIntyre J, et al. Role of low-frequency HIV-1 variants in failure of nevirapine-containing antiviral therapy in women previously exposed to single-dose nevirapine. Proc Natl Acad Sci USA 2011;108(22):9202-7.
[20] Li JZ, Paredes R, Ribaudo HJ, Svarovskaia ES, Metzner KJ, Kozal MJ, et al. Low-frequency HIV-1 drug resistance mutations and risk of NNRTI-based antiretroviral treatment failure: a systematic review and pooled analysis. JAMA 2011;305 (13):1327-35

[21] Cozzi-Lepri A, Noguera-Julian M, Di Giallonardo F, Schuurman R, Daumer M, Aitken S, et al. Low-frequency drug-resistant HIV-1 and risk of virological failure to first-line NNRTI-based ART: a multicohort european case-control study using centralized ultrasensitive 454 pyrosequencing. J Antimicrob Chemoth 2015;70 (3):930-40.

[22] Goodman DD, Zhou Y, Margot NA, McColl DJ, Zhong L, Borroto-Esoda K, et al. Low level of the K103N HIV-1 above a threshold is associated with virological failure in treatment-naive individuals undergoing efavirenz-containing therapy. AIDS 2011;25(3):325-33.

[23] Johnson JA, Li JF, Wei X, Lipscomb J, Irlbeck D, Craig C, et al. Minority HIV-1 drug resistance mutations are present in antiretroviral treatment-naive populations and associate with reduced treatment efficacy. PLoS Med 2008;5(7):e158.

[24] Simen BB, Simons JF, Hullsiek KH, Novak RM, Macarthur RD, Baxter JD, et al. Lowabundance drug-resistant viral variants in chronically HIV-infected, antiretroviral treatment-naive patients significantly impact treatment outcomes. J Infect Dis 2009;199(5):693-701.

[25] Inzaule SC, Hamers RL, Noguera-Julian M, Casadella M, Parera M, Kityo C, et al. Clinically relevant thresholds for ultrasensitive HIV drug resistance testing: a multi-country nested case-control study. Lancet HIV 2018;5(11):e638-e46.

[26] Halvas EK, Wiegand A, Boltz VF, Kearney M, Nissley D, Wantman M, et al. Low frequency nonnucleoside reverse-transcriptase inhibitor-resistant variants contribute to failure of Efavirenz-containing regimens in treatment- experienced patients. J Infect Dis 2010;201(5):672-80.

[27] Metzner KJ, Scherrer AU, von Wyl V, Boni J, Yerly S, Klimkait T, et al. Limited clinical benefit of minority K103N and Y181C-variant detection in addition to routine genotypic resistance testing in antiretroviral therapy-naive patients. AIDS 2014;28(15):2231-9.

[28] Messiaen P, Verhofstede C, Vandenbroucke I, Dinakis S, Van Eygen V, Thys K, et al. Ultra-deep sequencing of HIV-1 reverse transcriptase before start of an NNRTIbased regimen in treatment-naive patients. Virology 2012;426(1):7-11.

[29] Nicot F, Saune K, Raymond S, Jeanne N, Carcenac R, Lefebvre C, et al. Minority resistant HIV-1 variants and the response to first-line NNRTI therapy. J Clin Virol 2015;62:20-4.

[30] Boltz VF, Bao Y, Lockman S, Halvas EK, Kearney MF, McIntyre JA, et al. Low-frequency nevirapine (NVP)-resistant HIV-1 variants are not associated with failure of antiretroviral therapy in women without prior exposure to single-dose NVP. J Infect Dis 2014;209(5):703-10.

[31] Panpradist N, Beck IA, Vrana J, Higa N, McIntyre D, Ruth PS, et al. OLA-simple: a software-guided HIV-1 drug resistance test for low-resource laboratories. EBioMedicine 2019. doi: 10.1016/j.ebiom.2019.11.002. 